\title{
A Survey of Black Hole Detection Techniques in WSNs
}

\author{
Sandeep Kumar ${ }^{1}$, Dr.Suman Sangwan ${ }^{2}$ \\ M.tech Student, Computer Science, DCRUST, Murthal, Sonepat, India ${ }^{1}$ \\ Assistant Professor, Computer Science, DCRUST, Murthal, Sonepat, India ${ }^{2}$
}

\begin{abstract}
Wireless Sensor Networks (WSN) is a trending technology now-a-days and has a wide range of applications such as battlefield surveillance, traffic surveillance, forest fire detection, flood detection etc. But wireless sensor networks are susceptible to a variety of potential attacks which obstructs the normal operation of the network. Black hole attack is one of severe security threat that affects the network from its normal functioning by maliciously advertising itself having shortest route to the destination and then drops all receiving packets. There are lots of mechanisms have been proposed to defend network from black hole attack, but none of the solution looks most promising to defend against black hole attack. So in this paper, we have surveyed and compared the existing solutions to black hole attacks on AODV protocol. Tabular representation of comparison depicts clear picture of these solutions.
\end{abstract}

Keywords: AODV, Black hole attack, IDS, Routing

\section{INTRODUCTION}

A Wireless Sensor Network (WSN) consists of large The remainder of the paper is structured as follows. In next number of sensor nodes working in cooperation manner to section we discuss about some constraints of WSN and gather the information from the monitoring region. fundamentals of security that are essential to be considered Generally WSN have little or no infrastructure. There are as key concepts before implementing any protocol. In two types of WSNs: structured and unstructured [1]. In Section 2, we discuss some of the possible attacks in unstructured WSN there are huge numbers of nodes WSN. Section 3 describes the Black hole attack in both deployed randomly to monitor the region. Due to reactive and proactive routing protocols. A review of unavailability of physical presence on the region, network existing techniques to handle black hole attack is presented maintenance activities are difficult. In a structured WSN, in section 4. In section 5, Comparison of discussed all the nodes are deployed in fixed and planned manner. techniques is performed. Finally, section 6 concludes the Positive point of a structured network is that fewer nodes paper and points out future research directions.

can be deployed and requires fewer maintenance and management cost. In a WSN the object performing task of

\section{CONSTRAINTS FOR WSNS}

sensing is called a sensor. Sensor nodes are low power In the wireless sensor network, sensors are organized into devices equipped with one or more sensors, processor, the specific configuration to satisfy the requirements of admemory, power supply, a radio, and an actuator [2]. A hoc applications. Unfortunately, the connectivity cannot variety of mechanical power, thermal sensor, biological, remain unchanging at any working time. The sensor chemical,optical sensor, and magnetic sensors can be network is a broadcast network in which any signal can be attached to enhance the power of sensor nodes [1]. Since captured by adversaries at any time. These features make the sensor nodes wireless ad-hoc sensor networks more vulnerable than have limited memory and are deployed in harsh wired networks [2].

environment and in difficult locations, radio transmitter is Resource Constraints:

implemented to transfer the collected data to base station. WSNs have many applications such as military target tracking and surveillance, disaster relief, health monitoring, environment exploration seismic sensing to measure the environment.

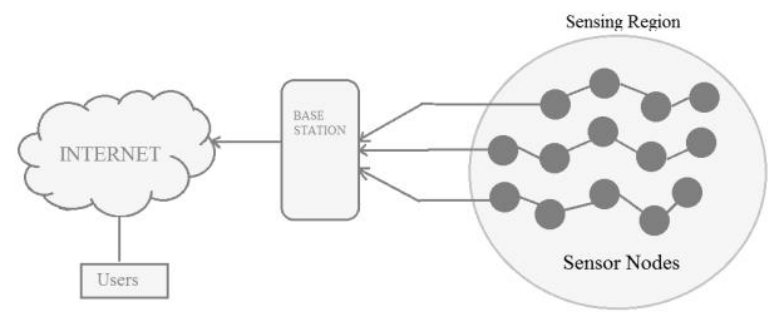

Figure 1.1 WSN model
Energy Constraints: Energy is one of the important constraints for WSNs. In sensor nodes energy consumption can be categorized in three parts: Sensor transducer, Communication among sensor nodes, microprocessor computation.

Memory Limitation: A sensor is a tiny device with a small amount of memory and storage space. Sensor nodes memory is usually includes flash memory and RAM (used for storing application programs, sensor info \& intermediate results of computations). Usually, there is not sufficient space to run complicated programs or codes after loading the OS and application code.

Lack of Central Control: Because of resource constraints and network dynamics it is not feasible to have a central 
point of control in sensor networks. Therefore security solutions must be decentralized and nodes must be able to achieve security [5].

Remote Locations: As sensor nodes are deployed in hardto-reach locations so it will be infeasible to continuously monitor and protect the nodes from attacks. That why it will be difficult maintain a secure network.

Error-prone Communication: Unreliable communication is a dangerous threat to sensor security. Packets in WSNs may be lost due to collision, channel errors or routing failures. This may interfere with security mechanisms.

\section{III.FUNDAMENTALS OF NETWORK SECURITY}

Computer and network security is the collection of all the malicious node could be to hinder the path-finding policies, mechanisms, and services that protect a computer process or to intercept all data packets being sent to the system or network from unauthorized access or unintended destination node. A more delicate form of this attack is use. So, to ensure Network as secure some security known as the grayhole attack, where the malicious node mechanisms are applied that are Non-repudiation, intermittently drops the data packets thereby making its Integrity, Availability, Privacy, Confidentiality, detection even more difficult [4].

Authorization, Authentication, Freshness[3].

\section{IV.ATTACKS IN WSN}

There are different kinds of attacks possible by malicious nodes to harm the network and make the network unreliable for communication and proper functioning. Some of such kinds of attacks are:

a) Jamming: Jamming attack is related with disrupting or interfering the radio frequencies used by sensor nodes. Attacker may get physical access to some nodes and creates jam in the network to disrupt the network. Jamming attack come under physical layer attack.

b) Tampering: Refers to gaining physical access to a set of sensors by tampering with their hardware configuration and making nodes to act as adversary node. Tampering is possible at physical layer.

c) Sybil Attack: Sybil attack is defined as a malicious device illegitimately taking on multiple identities. In Sybil attack an adversary can appear to be in multiple places at the same time. A single node presents multiple identities to other nodes in the sensor network either by fabricating or stealing the identities of authenticated nodes. It is a Network layer attack.

d) Wormhole attack: Wormhole attack is a critical attack in which the attacker records the packets (or bits) at one location in the network and tunnels those to another location. This generates a false scenario that the original sender is in the neighbourhood of the remote location. The tunnelling procedure forms wormholes in a sensor network. The tunneling or retransmitting of bits could be done selectively.

e) Hello Flood Attack: Hello flood attack uses HELLO packets as a weapon to convince the sensors in WSN. In this type of attack an attacker with a high radio transmission range (termed as a laptop-class attacker) and processing power sends HELLO packets to a number of sensor nodes which are dispersed in a large area within a WSN

Black hole: In Black hole attacks, a malicious node detects black hole attack but also improves the overall acts as a black hole to attract all the traffic in the performance. Limitation is that it cannot prevent the 
network from co-operative black hole attack because of assumption that black hole node cannot work in group.

SwarnaliHazra et.al. [5] proposed a trusted on-demand routing approach to prevent black hole attack depending on their trust model with different levels of trust computations. In this approach, black hole attackers are identified and isolated on context of data forwarding.

Fei Shi et.al [6] provides a cluster-based scheme form preventing black hole attacks in MANETs. It first employee's a powerful analytic hierarchy process (AHP) methodology to elect cluster heads $(\mathrm{CHs})$. Then $\mathrm{CHs}$ are well.

required to implement the black hole attack prevention scheme to not only detect the existence of black hole attacks but also identify the black hole nodes. Positive point with this scheme is that it is feasible and efficient in preventing black hole attacks.

R. TANUJA et al. [7] this article propose technique to eliminate Black Hole and False Data Injection attacks initiated by the compromised inside nodes and outside malicious nodes respectively using a new acknowledge scheme with low overhead. Advantage with this scheme is that it can successfully identify and eliminate $100 \%$ black hole nodes and ensures more than $99 \%$ packet delivery with increased network traffic.

Harsh Pratap Singh and Rashmi Singh [8] has proposed broadcast synchronization (BS) and relative distance (RD) method of clock synchronization which is used to prevent the black hole nodes. BS (Broadcast Synchronization) is very famous technique for clock synchronization process in Mobile-ad hoc Network. This paper has BS technique for removal of cooperative black hole attack. Sometimes the detection process for worms is failed in the clock synchronization. In this case this paper imposed another method for black hole detection using Relative Velocity distance.

Ming-Yang Su[9] in this several IDS (intrusion detection system) nodes are deployed in MANETs in order to detect and prevent selective black hole attacks. The IDS
nodes must be set in sniff mode in order to perform the socalled ABM (Anti-Black hole Mechanism) function, which is mainly used to estimate a suspicious value of a node according to the abnormal difference between the routing messages transmitted from the node. When a suspicious value exceeds a threshold, an IDS nearby will broadcast a block message, informing all nodes on the network, asking them to cooperatively isolate the malicious node. This study employs ns 2 to validate the effect of the proposed
IDS deployment, as IDS nodes can rapidly block a IDS deployment, as IDS nodes can rapidly block a
malicious node, without false positives, if a proper threshold is set. Advantage with this is that it is multipath passed protocol and packet loss rate can be decreased to
$11.28 \%$ and $14.76 \%$. Drawbacks: Failed at co-operative black hole attack detection. Muhammad Raza et.al [10] They have proposed a novel Anishi Gupta [17] Proposed a new method
architecture of FRIMM (A Forced Routing Information MEAODV(Modified Enhanced AODV), based on Modification Model) prevents black hole attacks in EAODV(Enhanced AODV).The MEAODV is based on wireless Ad Hoc network by introducing automatic error route discovery process for mitigating black hole effect. It correction in routing information that leads the node to does not have any overhead to the network. The similar select correct path thus secure transmission will take place logic is used as in EAODV but has few different condition between source and destination.

Copyright to IJARCCE

DOI 10.17148/IJARCCE.2015.45119
NeelamKhemariya et.al [11] have proposed an algorithm and it is implemented on AODV (Ad hoc on demand istance Vector) Routing protocol. The algorithm can the single Black hole attack and the dentify black hole nodes from the network and remove their entries from the routing table. The advantage of the algorithm is that it not only detects the black hole nodes in case when the node is not idle but it can also SubhashisBanerjee,MousumiSardar et.al[12] have proposed trust based mechanism for detection and mitigation of black hole nodes from the network. They ve introduced mechanism which detects malicious nodes the network without introducing additional control packets and without modifying routing table. Detection is iginator initiated hence there is no need to rely on mediate nodes. Trust mechanism

KashifSaghar et.al[13] have proposed RAEED (Robust formally Analyzed protocol for wireless sensor networks Deployment), which is able to address the problem of lack hole attacks using formal modeling and proves that

SatyajayantMisra et.al[14] have propose an efficient technique that uses multiple base stations deployed in the etwork to counter the impact of black holes on data ssion their work is based on how to deploy the base deployed in hostile environment. Simulation shows pack delivery ratio was $99 \%$ and detection rate was $100 \%$ but no. of base stations was not optimal.

Sonika Malik et.al [15] Have proposed the solution to black hole attack by using data routing table that stores routing information of neighbor nodes. This analyze the data routing table of nodes and send check packet to the neighbor nodes to get the information about nodes and rom this information they finds the trust worthy and able nodes and eliminate the malicious black hole malicious nodes.

Anurag Gupta et.al[16] Have proposed the solution to avoid denial of service and black hole attack in mobile adhoc network. In this the solution to detect the malicious node has been presented, for that all nodes in the network node and any misbehavior is detected by using RREQ
no time, Current time, Expire time Source sequence number and Destination Sequence number. Malicious nodes are dded to malicious list and when session expires malicious es are removed from malicious list because they assume that after some time malicious node stops doing malicious activity

parameters for checking the RREP message for better 
route discovery mechanism. Performance is compared \%.MDSR has less end to end delay as compared to DSR with EAODV and performance delivery ratio is slightly protocol.

higher. But negative point is that it does not consider co- $\quad$ S. Vidhyaand T. Sasilatha [23] proposed a black hole operative Black hole attack.

detection scheme in wireless sensor network by adding

H.Shafieiet.al[18] proposed two techniques to detect energy to sensor nodes externally through batteries that sinkholes in the network. In the first approach, base station increases network lifetime. The author provided a solution samples the residual energy of sensing nodes deployment to black hole attack by a public key encryption through region using a geostatistical method and estimates a Message Digest MD5 cryptographic function with 128 parameter called statistical estimator. Base station utilizes bithash value. While relaying messages from source to this parameter to estimate the presence of energy holes in destination, confidentiality, authenticity and integrity of deployment region using geostatistical frailty survival data packets is to be kept in mind. Nodes are in network in model. Energy holes around the base station are neglected such a way that a node acting as a mobile agent monitors whereas presence of energy holes in rest of the network the activities of neighboring nodes and informs trust ensures occurrence (presence/ existence) of sinkholes in manager about any changes in status of nodes. Trust network. Base station then instructs all of network nodes to manager verifies identity of each node in the network and avoid the suspicious region in their routing to mitigate the intimates to neighbors about the malicious behavior of a attack or ignore it. Second approach is Distributed node if it finds any to keep the network safe. MD5 marks a monitoring method comprising two phases: Distributed node malicious if it uses another node's signature and residual energy query phase and Distributed estimation packets are forwarded to neighbors through alternate route. and detection phase. Distributed monitoring method Providing energy externally increases network lifetime, detects region with lower average residual energy level packet delivery ratio, as well as throughput.

and applies a mitigation method to eliminate sinkholes.

N. Chaudhary and L. Tharani [24] proposed a Timer

S. S. Bajwa and M. K. Khan [19] proposed Grouped based detection mechanism to detect and eliminate black Black Hole Attack Security Model (GBHASM) to prevent hole nodes launched over AODV in mobile adhoc grouped black hole attacks in Ad hoc On-demand Distance networks. This scheme utilizes a trust value defined by vector (AODV) protocol in wireless ad hoc networks. This every node on its neighbors. Initially all neighbors are model is based on two modules. First module describes assigned max_trust value and a timer is set with each data how a new node becomes member of network. After packet. A node does not communicate with its neighbor if having joined the network, this node is assigned node code neighbor's trust value is less than min trust. The node (NC) pkk1 and pkk2. When node requests for shortest path checks by listening to wireless transmission whether have to destination with a packet having pkk2, then each node been received by next hop before timer expires. If node matches Node Code pkk1 with pkk2. If they match within could not hear wireless transmission of next hop, it reduces Time to Live (TTL), routing information is shared with trust value of next hop and broadcasts this information to intermediate node otherwise packet is forwarded to next all nodes in network so that they can update their routing node. This model has low delay and high performance.

tables. If node's next hop continues to drop packets, its

Varshney et al., [20] proposed a monitoring method trust value goes on decreasing and becomes less than called Watchdog AODV mechanism to form detect black min_trust. All nodes in network put such a node in their hole nodes in mobile adhocnetworks.In this method nodes blacklist table. In this way, all blackhole nodes get act as watchdogs monitor their neighbors locally using eliminated from the network. Packet delivery ratio gets control messages by listening to all nodes within improved as black hole nodes are detected and removed transmission range to detect misbehaving as well as from the network.

black hole node. Black hole node once detected, is Siddiqua et al., [25] proposed a secure knowledge excluded from the path of transmitting messages. algorithm to detect and mitigate black hole attack on Limitation of Watchdog AODV is that it is vulnerable to AODV by taking packet drop reasons into consideration attack of two consecutive nodes. It can monitor only first before declaring a trusted node as black hole node. Each node while the consecutive node performs attack. node monitors the behavior of its neighbor by listening to Watchdog AODV has higher packet delivery ratio and packet transmission wirelessly. Every node compares the lower overhead than AODV. neighbor information with its knowledge table M. Mohanpriya, I. Krishanamurthi [21] presented information. The nodes monitor the control packets as well Modified Dynamic Source Routing protocol (MDSR) to as data packets to prevent selective dropping. When packet detect and prevent selective black hole attack by analyzing dropping reaches to a threshold then before declaring a forwarding behavior of nodes. This approach detects the node to be malicious the algorithm first checks whether presence of gray hole attackers in source route based on suspected node is destination or not. It also checks packet difference between number of packets source node sends drop reasons such as Time to live (TTL) and residual and number of packets that are actually received by energy. If suspected node is detected to be a black hole, its destination. IDS nodes deployed in network broadcast the id is broadcasted to all other nodes in network so that block message to all nodes and then suspected malicious malicious node can be avoided in routing process. Secure nodes are isolated from the routing path as well as AODV shows better performance in terms of throughput network. Advantages of MDSR are that it reduces packet and delay as compared to existing AODV.

drop ratio by $64 \%$ but increased overhead ratio by 8 


\section{COMPARISON}

Various techniques are discussed based on various criteria, [1] which are base routing protocol used, Modifies routing table or not, new control packets introduced or not, type of black hole detected and simulation tool used.

\begin{tabular}{|c|c|c|c|c|c|c|c|}
\hline $\begin{array}{l}\text { Sf Tedinigut } \\
\text { No. }\end{array}$ & $\begin{array}{l}\text { Roth } \\
\text { Proto } \\
\text { Lsed }\end{array}$ & 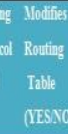 & 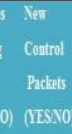 & SoOL & 1. Performance Whatices & Restls & Year \\
\hline 1. $\mathrm{NRM}[4]$ & $\mathrm{AOD}$ & YES & YES & NS2 & $\begin{array}{l}\text { Routing overhead, PDR, } \\
\text { CPU usage, Nemory usage, } \\
\text { Delay }\end{array}$ & Netrookis Performance lmproved & 2012 \\
\hline 2. CST-AODV[5] & $A O D$ & V YES & YES & . & Packetloss Rate & Packet Loss Rate are negligible & 2014 \\
\hline 3. Cluster Based [6] & $A O D$ & YES & No & NS2 & PDR vistrafficload & $\begin{array}{l}\text { ImprovedPacket defreryyabo under } \\
\text { black hole antad. }\end{array}$ & 2013 \\
\hline 4. $\mathrm{BHnFDLA}[7]$ & MAC & so & YES & MATLAB & PDR vis compromisednodes & $\begin{array}{l}\text { es Give } 100 \% \text { packe filtering efffiency } \\
\text { and } 99 \% \text { padert deliveryratio }\end{array}$ & y 2013 \\
\hline 5. Secure Path Based $[8]$ & OLSR & YES & YES & NS2 & $\begin{array}{l}\text { Throughpput end to end delays, } \\
\text { PDR, Iiter }\end{array}$ & $\begin{array}{l}\text { ys. Effective peffomancein tems of PDR, } \\
\text { Throushppit, end to end delay }\end{array}$ & R, 2014 \\
\hline 6. DS Based[9] & AOD & $V$ YES & YES & N52 & Total packetloss rate & $\begin{array}{l}\text { Packetloss reduced to } 10.05 \% \text { and } \\
\text { detection rate } 100 \%\end{array}$ & 2011 \\
\hline 7. FRDa[10] & AOD & $V$ YES & No & - & $\cdot$ & - & 2011 \\
\hline 8. Neelametal[11] & $A O D$ & so & YES & NS2 & $\begin{array}{l}\text { Throughput, PDR, endito and } \\
\text { delay }\end{array}$ & gd Improvenentwolippefomance & 2013 \\
\hline 9. Tnut Based[12] & AOD & $v$ so & No & - & - & - & 2013 \\
\hline 10. RAEED [13] & [NSE. & NS YES & so & TOSSIM & \%of nodes blodked & Robust and lower ovethead & 2014 \\
\hline 11. BAMBi[14] & - & so & No & $\begin{array}{l}\text { Realisic } \\
\text { simulation }\end{array}$ & $\begin{array}{l}\text { Packet delivery suceess, } \\
\text { packet deivery fialure }\end{array}$ & $\begin{array}{l}\text { Packet delivery ratio is } 99 \% \text { Detection } \\
\text { rafio is } 100 \%\end{array}$ & n 2011 \\
\hline 12. Alamm Based[15] & AODV & YES & YES & NS2 & $\cdot$ & $\cdot$ & \\
\hline 13. DSS Bassed[16] & AODV & YES & YES & NS2 & Throughpurt, end to end dedy & Better trroughput and end to end delay & 2015 \\
\hline 14. IEAODV[17] & AODV & YES & YES & NS2 & $\begin{array}{l}\text { PDR, end to end delay, no. } \\
\text { of malicious nodes. }\end{array}$ & Better PDR as compared to EAODV & 2013 \\
\hline 15. Geostatistical based[18] & AODV & so & so & OLAET- & False positive false negative & Adopted $95 \%$ confidence level & 2014 \\
\hline 16. GBHASM[19] & AODV & YES & YES & NS2 & No. of RREQ and RREP & High perfomanceless delay & 2010 \\
\hline 17. WatchdogAODV[20] & AODV & YES & YES & NS2 & $\begin{array}{l}\text { PDR, MACload, end to end } \\
\text { delay }\end{array}$ & Improved PDR andend to end delay & 2014 \\
\hline 18. IDSR[21] & DSR & YES & YES & GLOMOSIM & $\begin{array}{l}\text { Packet trop ratio, end to end } \\
\text { delay, PDR, ovehend }\end{array}$ & Percentage of Padcelloss ratei is better & 2013 \\
\hline 19. ACK based[22] & AODV & so & YES & OPNET & & & 2014 \\
\hline 20. $\mathrm{ID} 5$ based[23] & AODV & so & so & vS2 & $\begin{array}{l}\text { PDR, Throughput, end to end } \\
\text { delay }\end{array}$ & Improved overall netw ark perfomance & 2014 \\
\hline 21. Timer based[24] & AODV & YES & YES & EXata-cyber & PDRv vno of attacker & ImprovedPDR & 2015 \\
\hline 22. Knowledge bsed[2] & AODV & YES & YES & NS2 & $\begin{array}{l}\text { PDR visno of malicious } \\
\text { nodes }\end{array}$ & Effectrie PDR & 2015 \\
\hline
\end{tabular}

\section{CONCLUSION}

Wireless Sensor Networks are vulnerable to many types of attacks due to deployment of sensor nodes in an unattended environment. These types of networks are suffered from the black hole attack as there is no centralized security management. This paper provided a survey on various countermeasures for black hole attack. In this survey, firstly we have given the security goals of a network. Next, we have presented some of the possible network layer attacks in WSNs. This survey also gives the tabular analysis of various security mechanisms to prevent network from black hole attack. It is to be believed that this survey will help future researches in developing a good knowledge about the attacks and their countermeasures.

\section{REFERENCES}

Di Pietro, R., S. Guarino, N. V. Verde, and J. DomingoFerrer, "Security in wireless ad-hoc networks-A survey," Computer Communications, Vol. 51, pp. 1-20, 2014

[2] Kifayat, Kashif, MadjidMerabti, Qi Shi, and David LlewellynJones. "Security in wireless sensor networks." In Handbook of Information and Communication Security, pp. 513-552. Springer Berlin Heidelberg, 2010.

[3] Shio Kumar Singh, M P Singh, D K Singh, "A Survey on Network Security and Attack Defense Mechanism For Wireless Sensor Networks", International Journal of Computer Trends and Technology- May to June Issue 2011.

[4] Arunmozhi, S. A., and Y. Venkataramani. "Black Hole Attack Detection and Performance Improvement in Mobile Ad-Hoc Network." Information Security Journal: A Global Perspective 21, no. 3 (2012): $150-158$

[5] ] Hazra, Swarnali, and S. K. Setua. "Blackhole Attack Defending Trusted On-Demand Routing in Ad-Hoc Network." In Advanced Computing, Networking and Informatics-Volume 2, pp. 59-66. Springer International Publishing, 2014.

[6] Shi, Fei, Weijie Liu, Dongxu Jin, and Jooseok Song. "A cluster-based countermeasure against blackhole attacks in MANETs." Telecommunication Systems 57, no. 2 (2014): 119-136.

[7] Tanuja, R., M. K. Rekha, S. H. Manjula, K. R. Venugopal, S. S. Iyengar, and L. M. Patnaik. "Elimination of black hole and false data injection attacks in wireless sensor networks." In Proceedings of the Third International Conference on Trends in Information, Telecommunication and Computing, pp. 475-482. Springer New York, 2013

[8] Singh, Harsh Pratap, and Rashmi Singh. "A mechanism for discovery and prevention of coopeartive black hole attack in mobile ad hoc network using AODV protocol." In Electronics and Communication Systems (ICECS), 2014 International Conference on, pp. 1-8. IEEE, 2014.

[9] Su, Ming-Yang. "Prevention of selective black hole attacks on mobile ad hoc networks through intrusion detection systems." Computer Communications 34, no. 1 (2011): 107-117

[10] Raza, Muhammad, and Syed IrfanHyder. "A forced routing information modification model for preventing black hole attacks in wireless Ad Hoc network." In Applied Sciences and Technology (IBCAST), 2012 9th International Bhurban Conference on, pp. 418422. IEEE, 2012.

[11] Khemariya, Neelam, and Ajay Khuntetha. "An Efficient Algorithm for Detection of Blackhole Attack in AODV based MANETs." International Journal of Computer Applications 66, no. 18 (2013).

[12] Banerjee, Subhashis, MousumiSardar, and KoushikMajumder. "AODV Based Black-Hole Attack Mitigation in MANET." In Proceedings of the International Conference on Frontiers of Intelligent Computing: Theory and Applications (FICTA) 2013, pp. 345-352. Springer International Publishing, 2014.

[13] Saghar, Kashif, David Kendall, and Ahmed Bouridane. "Application of formal modeling to detect black hole attacks in wireless sensor network routing protocols." In Applied Sciences and Technology (IBCAST), 2014 11th International Bhurban Conference on, pp. 191-194. IEEE, 2014.

[14] Misra, Satyajayant, KabiBhattarai, and GuoliangXue. "BAMBi: blackhole attacks mitigation with multiple base stations in wireless sensor networks." InCommunications (ICC), 2011 IEEE International Conference on, pp. 1-5. IEEE, 2011.

[15] Malik, Sonika, and InduKashyap. "Identifying, Avoidance and Performance Assessment of Black Hole Attack on AODV Protocol in MANET." International Journal of Computer Applications 95, no. 17 (2014): 6-11.

[16] Gupta, Anurag, Bhupendra Patel, Kamlesh Rana, and Rahul Pradhan. "Improved AODV Performance in DOS and Black Hole Attack Environment." In Computational Intelligence in Data Mining-Volume 2, pp. 541-549. Springer India, 2015.

[17] Gupta, Anishi. "Department of Computer of Engineering, Delhi Technological University, New Delhi, India." In Computational Intelligence and Computing Research (ICCIC), 2013 IEEE International Conference on, pp. 1-6. IEEE, 2013.

[18] Taylor, Vincent F., and Daniel T. Fokum. "Mitigating black hole attacks in wireless sensor networks using node-resident expert 
systems." In Wireless Telecommunications Symposium (WTS), 2014, pp. 1-7. IEEE, 2014.

[19] Bajwa, ShahidShehzad, and Muhammad Khalid Khan. "Grouped Black hole Attacks Security Model (GBHASM) for Wireless AdHoc Networks." In Computer and Automation Engineering (ICCAE), 2010 The 2nd International Conference on, vol. 1, pp. 756-760. IEEE, 2010

[20] Varshney, Tarun, Tushar Sharma, and Pankaj Sharma "Implementation of Watchdog Protocol with AODV in Mobile Ad Hoc Network." In Communication Systems and Network Technologies (CSNT), 2014 Fourth International Conference on, pp. 217-221. IEEE, 2014.

[21] Mohanapriya, M., and IlangoKrishnamurthi. "Modified DSR protocol for detection and removal of selective black hole attack in MANET." Computers \& Electrical Engineering 40, no. 2 (2014): 530-538.

[22] Baadache, Abderrahmane, and Ali Belmehdi. "Struggling against simple and cooperative black hole attacks in multi-hop wireless ad hoc networks." Computer Networks 73 (2014): 173-184.

[23] S. Vidhyaand T. Sasilatha, "Performance Analysis of Black Hole Attack DetectionScheme using MD5 Algorithm in WSN ", IEEE International Conference on Smart Structures \& Systems (ICSSS2014), Chennai-INDIA. pp. 51-54

[24] N. Chaudhary and L. Tharani, " Preventing Black Hole Attack in AODV using Timer-Based Detection Mechanism", SPACES-2015, Dept of ECE, K L UNIVERSITY, 2015, pp. 1-4

[25] Siddiqua et al., "Preventing Black Hole Attacks in MANETs Using Secure Knowledge Algorithm", SPACES-2015, Dept of ECE, K L UNIVERSITY, 2015, pp. 421-425. 